\section{Is day-1 postoperative review necessary after pars plana vitrectomy?}

P Alexander, L Michaels and R Newsom

\author{
Abstract
}

Purpose This study aimed to determine the proportion of patients requiring alteration in management based on the findings of the day-1 postoperative visit after pars plana vitrectomy, and to identify clinical characteristics that predict the need for unexpected intervention.

Patients and methods A retrospective case note review was conducted of all patients who underwent pars plana vitrectomy and who then attended for review on the first postoperative day. All patients received routine prophylactic anti-glaucoma medication.

Results Two hundred and seventy-three patients examined on day 1 following vitrectomy were studied. Indications for surgery included retinal detachment, epiretinal membrane, macular hole, vitreous haemorrhage, diabetic eye disease, and floaters. Twenty-gauge (20G) vitrectomy was performed in 124 eyes (45\%); 23-gauge (23G) vitrectomy was performed in 149 eyes (55\%). Phacoemulsification was performed concurrently in 51/273 (19\%) eyes. Ten patients $(3.7 \%)$ required unexpected intervention on day 1 owing to intraocular pressure (IOP) $>30(2 / 273)$, IOP $<6(5 / 273)$, or unexpected return to theatre for anterior chamber washout $(3 / 273)$. There was no difference in intervention rate or day-1 IOP between 20G and 23G cases. Hypotony was less common if gas tamponade was used $\left(\chi^{2}\right.$-test, $\left.P<0.001\right)$. Patients undergoing combined phacoemulsification and 20G vitrectomy were significantly more likely to require intervention on day 1 than patients undergoing 20G vitrectomy alone (15.0 vs $1.9 \%, P=0.029$, Fisher's exact test) but this was not the case for patients undergoing $23 \mathrm{G}$ vitrectomy ( 0 vs $4.2 \%$, Fisher's exact test, $P=0.58)$.

Conclusions The intervention rate on the first day after vitrectomy is low and day-1 postoperative review can be safely omitted in the majority of patients undergoing vitrectomy.

Eye (2015) 29, 1489-1494; doi:10.1038/eye.2015.134;

published online 28 August 2015

Introduction

In 1988, Isernhagen et $a l^{1}$ found that a high proportion $(52 \%)$ of vitrectomy patients require inpatient treatment in the immediate postoperative period. This finding has dictated the current standard of care, which suggests that best clinical practice must include a postoperative first day review for all patients undergoing vitreoretinal (VR) surgery. However, of the postoperative events cited by Isernhagen et $a l^{1}{ }^{1}$ the majority were side effects of general anaesthesia rather than ocular surgical complications. With increasing use of local anaesthesia in VR surgery, the use of smallergauge vitrectomy instruments and optimisation of the postoperative treatment regimen, postoperative events following uncomplicated surgery have become uncommon. ${ }^{2-4}$ The necessity and benefits of day- 1 postoperative review after routine vitrectomy remain the subject of debate. Day-1 review has an associated economic cost and is also inconvenient for patients, who are often forced to travel long distances to access a specialist VR service. $^{5}$

The demand for VR surgery is growing. The numbers of pars plana vitrectomies performed in National Health Service hospitals in England has increased by an average of $14 \%$ each year over the last 6 years. ${ }^{6,7}$ The resulting pressure on National Health Service resources has resulted in considerable variation in practice between centres, and a recent survey of UK VR surgeons has found that up to $37 \%$ of respondents have abandoned the day- 1 postoperative review. ${ }^{8}$

The main concern on the first postoperative day is the intraocular pressure (IOP). The reported incidence of high IOP at day 1 varies between 6 and $67 \% .^{9-12}$ However, numerous
Department of Ophthalmology, University Hospital Southampton, Southampton, UK

Correspondence:

P Alexander, Department of Ophthalmology, University Hospital Southampton, Tremona Road, Southampton, Hampshire SO16 6YD, UK

Tel: +44 (0)23 8120 4422;

Fax: +44 (0)23 81206614 .

E-mail: p.alexander@soton. ac.uk

Received: 19 November 2014

Accepted in revised form: 14 May 2015

Published online:

28 August 2015

This work has been partially presented at the 2013 annual meeting of the British and Eire Association of Vitreoretinal Surgeons (BEAVRS), Bristol, UK, and at the Royal Society of Medicine Annual Student and Trainee Ophthalmology Conference (ASTOC) (Poster Prize). 
groups have suggested that prophylactic topical treatment with apraclonidine ${ }^{13}$, dorzolamide, and/or timolol $^{14}$ are effective at preventing IOP spikes.

We are a tertiary referral centre within a large teaching hospital on the South Coast of England. In our department, $95 \%$ of pars plana vitrectomy patients have day-case surgery under local anaesthesia. All patients, unless contraindicated, receive prophylactic treatment to prevent an IOP spike. Patients are asked to return for examination the following day; however, those who have travelled long distances are asked to attend their local eye unit for day- 1 assessment rather than returning to our centre. The aims of this study were to determine the proportion of vitrectomy patients requiring an alteration in management based on the findings of the day- 1 postoperative visit, and to identify clinical characteristics that predict the need for this unexpected intervention.

\section{Materials and methods}

A retrospective analysis of patients managed by the VR service at the University Hospital Southampton (UHS) was conducted. The Medisoft electronic database was used to compile a consecutive series of patients who underwent pars plana vitrectomy under the care of a single consultant (RSN) team between December 2010 and April 2014, and attended for outpatient review on the first postoperative day. Patients were operated by a consultant, fellow, or senior registrar in VR surgery. Until April 2013, pars plana vitrectomy was performed using 20-gauge (20G) instruments and semi-sutureless tunnelled incisions. The infusion port was left unsutured and each of the remaining two ports were sutured with a single 8.0 vicryl suture through both the sclera and conjunctiva. After April 2013, all vitrectomy patients received 23-gauge (23G) surgery and ports were sutured only if they were leaking at the end of surgery.

In all patients undergoing combined phacovitrectomy, phacoemulsification and intraocular lens implantation was performed before the vitrectomy, through a superior corneal tunnel. Both procedures were performed by the same surgeon. For 23G surgery, scleral ports were pre-placed before the phacoemulsification procedure started.

Retinal breaks were treated either with trans-scleral cryotherapy or with endolaser retinopexy. For patients requiring gas tamponade, isovolumetric concentrations were used (ie, $20 \% \mathrm{SF}_{6}, 16 \% \mathrm{C}_{2} \mathrm{~F}_{6}$, or $14 \% \mathrm{C}_{3} \mathrm{~F}_{8}$ ). Silicone oil tamponade (either 1000 cs or 5700 cs) was used when deemed necessary by the operating surgeon. Eyes not requiring specific tamponade were filled with balanced salt solution (BSS) for 20G vitrectomy cases or air for $23 \mathrm{G}$ cases.
All patients received $500 \mathrm{mg}$ of oral acetazolamide immediately after the procedure, and commenced a 7-day course of acetazolamide $250 \mathrm{mg}$ slow-release twice daily and were advised to take the first dose before attending the day-1 review. All patients also received a 1-month course of guttae timolol $0.25 \%$ twice daily, unless contraindicated, in which case an alternative topical antiglaucoma treatment was prescribed. Topical dexamethasone and chloramphenicol drops were also prescribed. Patients were advised to start the topical treatment only after the clinical assessment on day 1 .

On the first postoperative day, all patients were examined by a consultant or senior clinical fellow. Slitlamp biomicroscopy and dilated fundoscopy were performed and IOP measurements were taken by Goldmann applanation. For the purposes of this study, clinically significant raised IOP was defined as Goldmann IOP $\geq 30 \mathrm{~mm} \mathrm{Hg}$.

Data collected on each patient included ocular comorbidity, indication for surgery, and any other procedures performed at the time of vitrectomy (eg, phacoemulsification, membrane peel, endolaser, and cryotherapy). The primary outcome was the proportion of patients requiring a change in management based on the findings of the day-1 examination.

\section{Statistical analysis}

Fisher's exact test $/ \chi^{2}$-test were performed to test for the statistical significance of data collected from each patient (eg, indication for surgery, type of tamponade, intraoperative complications, and concurrent phacoemulsification) in predicting change of management (SPSS Statistics v21, IBM Corp, Armonk, NY, USA). To calculate proportions, their differences and the confidence interval (CI) for the difference were used (Confidence Interval Analysis (CIA) Software Version: 2.2.0, Trevor Bryant, Southampton, UK). $P$-values $<0.05$ were considered statistically significant.

\section{Results}

A total of 275 patients who underwent vitrectomy under the care of the surgical team during the study period returned for follow-up at UHS on the first postoperative day. Two of these patients were excluded, because they had undergone therapeutic vitrectomy for acute endophthalmitis, and daily review for such patients is widely accepted and not a matter of dispute. A total of 273 patients were included in the study. Of these, 124 patients underwent $20 \mathrm{G}$ surgery and 149 patients underwent 23G surgery. Baseline characteristics of both groups of patients are shown in Table 1. No patients required inpatient care or any emergency advice or 
Table 1 Patient demographics

\begin{tabular}{lccc}
\hline & $\begin{array}{c}20 \text { Gauge } \\
\mathrm{n}=124\end{array}$ & $\begin{array}{c}23 \text { Gauge } \\
\mathrm{n}=149\end{array}$ & P-value \\
\hline Mean age (SD) & 60.3 years (15.9) & 61.9 years $(15.6)$ & 0.397 \\
Male (\%) & $67(54.0 \%)$ & $70(47.0 \%)$ & 0.275 \\
Local Anaesthetic (\%) & $119(96.0 \%)$ & $140(94.0 \%)$ & 0.585 \\
Pre-existing & $4(3.2 \%)$ & $9(6.0 \%)$ & 0.394 \\
glaucoma (\%) & & & \\
& & & \\
Pre-operative lens status (\%) & & 32 & 0.282 \\
$\quad$ Pseudophakic & 20 & 117 & 0.3611 \\
Phakic & 103 & 0 & 0.454 \\
$\quad$ Aphakic & 1 & & \\
\hline
\end{tabular}

There was no statistically significant difference preoperatively between patients undergoing 20-gauge and 23-gauge vitrectomy surgery.

Table 2 Indications for pars plana vitrectomy

\begin{tabular}{lc}
\hline Indication for pars plana vitrectomy & No. of patients $(\mathrm{n}=273)$ \\
\hline Retinal detachment & 137 \\
Macular hole & 33 \\
ERM & 32 \\
New vitreous haemorrhage & 28 \\
Vitreous floaters & 11 \\
Diabetic eye disease & 7 \\
Silicone oil in vitreous cavity & 6 \\
Dropped nucleus & 5 \\
IOL in vitreous cavity & 5 \\
Submacular haemorrhage & 3 \\
Surgical capsulotomy & 2 \\
Heavy liquid in situ & 2 \\
Vitritis & 2 \\
\hline
\end{tabular}

intervention overnight. Indications for vitrectomy surgery are shown in Table 2. Fifty-one (19\%) patients required concurrent phacoemulsification, either as part of macular hole surgery or because of cataract that was sufficiently dense as to preclude an adequate fundal view during vitrectomy. Eleven patients had iatrogenic retinal tears that were identified at the time of surgery. Type of tamponade used is shown in Table 3.

Of 273 patients, the day- 1 postoperative examination culminated in an unexpected intervention in 10 patients $(3.7 \%)$. A change in medication was required in $6 / 273$, either owing to IOP > 30 (2/273) or hypotony $(4 / 273)$. Unexpected return to theatre was required in $4 / 273$. Indications included flat anterior chamber (AC) requiring reinflation (1/273), hyphaema affecting $2 / 3$ of the AC $(1 / 273)$, retained AC nuclear fragment $(1 / 273)$, and intraocular foreign body (1/273). The foreign body appeared to be a cotton fibre from a surgical swab. No patients reported side-effects from acetazolamide that warranted discontinuing this treatment. A summary of all patients requiring unexpected intervention on day 1 is shown in Table 4.
Table 3 Type of tamponade used

\begin{tabular}{lc}
\hline Tamponade & No. of patients $(\mathrm{n}=273)$ \\
\hline None (ie, balanced salt solution) & 27 \\
Air & 50 \\
$\mathrm{SF}_{6}$ & 58 \\
$\mathrm{C}_{2} \mathrm{~F}_{6}$ & 61 \\
$\mathrm{C}_{3} \mathrm{~F}_{8}$ & 19 \\
Silicone oil (1000 cs or $5700 \mathrm{cs})$ & 56 \\
Perfluorodecalin (for 1 week only) & 2 \\
\hline
\end{tabular}

Rate of intervention was not associated with indication for surgery $(P=0.83)$, gauge of surgery $(P=1.00)$, or type of tamponade $(P=0.51)\left(\chi^{2}\right.$-tests $)$. Patients undergoing $20 \mathrm{G}$ vitrectomy combined with phacoemulsification were significantly more likely to require intervention on day 1 than patients undergoing 20G vitrectomy alone (15.0 vs $1.9 \%$, difference $=13.1 \%$; $95 \% \mathrm{CI}=2.2-34.2 \%, P=0.029$, Fisher's exact test). This was not the case for those patients undergoing $23 \mathrm{G}$ vitrectomy combined with phacoemulsification (0 vs $4.2 \%$, Fisher's exact test, $P=0.58)$.

There was no significant difference in mean IOP on day 1 between the 20G (14.73 $\mathrm{mm} \mathrm{Hg}(\mathrm{SD} 6.7)$ ) and 23G subgroups (13.81 $\mathrm{mm} \mathrm{Hg}(\mathrm{SD} 5.8))(P=0.24)$. Neither the gauge of surgery nor the indication for surgery was predictive of hypotony (IOP $<6 \mathrm{~mm} \mathrm{Hg}$ ), high IOP $(>21 \mathrm{~mm} \mathrm{Hg})$, or very high IOP $(>30 \mathrm{~mm} \mathrm{Hg})\left(\chi^{2}\right.$ $P=0.2)$. The rate of hypotony on day 1 was lower in the eyes that received gas tamponade $\left(\mathrm{SF}_{6}, \mathrm{C}_{2} \mathrm{~F}_{6}\right.$, or $\left.\mathrm{C}_{3} \mathrm{~F}_{8}\right)$ than if BSS, air, or silicone oil were used (2.2 vs 12.4\%, difference $=10.2 \% ; 95 \% C I=3.9-17.4 \%, P=0.001, \chi^{2}$-test).

\section{Discussion}

This study shows that on the first day after pars plana vitrectomy, $3.7 \%$ of patients had clinical findings significant enough to require a change in management. This low intervention rate is in stark contrast to a previous study, published more than 25 years ago, which found that $52 \%$ of patients required some form of unexpected intervention in the immediate postoperative period and has defined the standard of care ever since, namely that all patients undergoing VR surgery should receive a postoperative day-1 examination.

A number of groups have investigated the importance of the clinical examination on the first postoperative day after vitrectomy surgery. Most of these studies have focussed only on the day-1 IOP, as this is the main concern in the immediate postoperative period, but these studies have produced conflicting results. Two studies have concluded that the day- 1 examination is important for the detection and treatment of the IOP spike. Lee et al ${ }^{15}$ found that $4.8 \%$ of 89 patients undergoing $20 \mathrm{G}$ vitrectomy 
Table 4 Patients requiring intervention based on the day-1 postoperative examination

\begin{tabular}{lcllcll}
\hline Indication & Gauge & Phaco & Tamponade & IOP & Reason for intervention & Rx change \\
\hline Epiretinal membrane & 20 & Yes & Air & Not recorded & AC fragment & AC washout required \\
Floaters & 20 & Yes & Air & 25 & Filaments in wound & AC washout required \\
Retinal detachment & 23 & No & $\mathrm{SF}_{6}$ & 33 & Hyphaema $(2 / 3$ of AC) & AC washout required \\
Retinal detachment & 20 & No & Oil & 35 & High IOP & Prescribed iopidine \\
Retinal detachment & 20 & No & $\mathrm{C}_{2} \mathrm{~F}_{6}$ & 30 & High IOP & Timolol $\rightarrow$ Combigan \\
Epiretinal membrane & 20 & Yes & None & 3 & Low IOP & Diamox and timolol stopped \\
Retinal detachment & 23 & No & Oil & 4 & Low IOP & Diamox and timolol stopped \\
Retinal detachment & 23 & No & Oil & 6 & Low IOP & Diamox stopped \\
Vitreous haemorrhage & 23 & No & Air & 0 & Low IOP & AC re-inflated \\
Vitreous haemorrhage & 23 & No & Air & 4 & Low IOP & Stop Diamox \\
\hline
\end{tabular}

require treatment on day 1 for IOP $>30 \mathrm{~mm} \mathrm{Hg}$, and that this group of patients were less likely to have a raised IOP at 2 weeks, compared with patients in whom the day-1 examination had been omitted (2.4 vs $8.5 \%$ ). In a study of day-1 postoperative IOP spikes following VR surgery, Wong et $a l^{5}$ found that $14 \%$ of 235 patients undergoing $20 \mathrm{G}$ had an IOP $>30 \mathrm{~mm} \mathrm{Hg}$ on day 1 that required treatment, and that patients with a preoperative IOP $>20 \mathrm{~mm} \mathrm{Hg}$ were significantly more likely to have an IOP > $30 \mathrm{~mm} \mathrm{Hg}$ postoperatively. Of note, however, prophylactic anti-glaucoma medication was not used in either of these two studies, despite evidence in the literature that topical agents can be effective at preventing, or at least attenuating, the IOP spike. ${ }^{13,14}$

In contrast, the study by Cochrane et al ${ }^{16}$ of 81 patients undergoing $23 \mathrm{G}$ vitrectomy advocates the abandonment of the day-1 visit. The authors checked the IOP immediately postoperatively and found no patients with an IOP $>30 \mathrm{~mm} \mathrm{Hg}$. At 2 weeks' review, 6 of 81 patients had an IOP $>30 \mathrm{~mm} \mathrm{Hg}$. The authors contend that a review on day 1 would not have prevented raised pressures and is therefore unnecessary, and that routine follow-up at 2 weeks is safe. However, Cochrane et al ${ }^{16}$ caution that patients with a known history of glaucoma would benefit from an earlier review. Russell-Hermanns et $\mathrm{al}^{17}$ found that patients receiving $\mathrm{SF}_{6}$ were at the risk of raised IOP on day 1 , but the day- 1 visit could be omitted in patients receiving air, silicone oil, or no tamponade.

All patients received oral acetazolamide immediately postoperatively and on the first postoperative day, before the follow-up appointment. No patients complained of side effects of acetazolamide, such as more frequent micturition or paraesthesia, at the day-1 postoperative visit. In our study, hypotony on day 1 was observed in five patients, but choroidal detachments, choroidal folds, and corneal decompensation were not observed in any of the five patients. One patient required reinflation of the AC. The other four patients were treated with an alteration in the treatment regimen. The IOP normalised in these four patients within $48 \mathrm{~h}$. Although the acetazolamide would have contributed to this low IOP, it seems unlikely that two doses of acetazolamide would be sufficient to cause hypotony, given the low incidence of hypotony in this study. There were no permanent sequelae of hypotony in any patients.

In our study, patients receiving $\mathrm{SF}_{6}, \mathrm{C}_{2} \mathrm{~F}_{6}$ or $\mathrm{C}_{3} \mathrm{~F}_{8}$ gas tamponade were less likely to have a low IOP on day 1 (2.2 vs $13.0 \%, \chi^{2}, P=0.001$ ) than if air, silicone oil or no tamponade were used, but were not more likely to develop raised IOP on day 1 .

In this study, risk of intervention was significantly higher for patients undergoing combined phacoemulsification and 20G vitrectomy than for 20G PPV alone (15 vs 1.9\% ( $P=0.028$, Fisher's exact test $)$ ). This was an unexpected finding, especially because two patients required AC washout for unusual complications. We are wary of drawing too many conclusions from a relatively small subgroup of our cohort, but nevertheless our results do suggest that day- 1 review still has value in this subset of patients. Patients undergoing 23G vitrectomy with phacoemulsification were not at increased risk of intervention compared with patients undergoing 23G vitrectomy alone ( 0 vs $4.2 \%, \chi^{2}, P=0.6$ ).

At $3.7 \%$, our intervention rate is low, but it is difficult to know how low the intervention rate needs to be before abandonment of the day- 1 visit will be considered a safe and good practice. However, there is precedence from cataract surgery that provides some guidance. During the transition of cataract surgery from extracapsular cataract extraction to phacoemulsification, it was noted that the day-1 postoperative intervention rate after routine phacoemulsification was around $3 \%$ and this was deemed sufficiently low to justify the omission of the day-1 visit. ${ }^{18-20}$ Allan et al ${ }^{18}$ suggest that if the intervention rate is $<5 \%$, the visit is probably unnecessary, apart from the mutual reassurance for the patient and the surgeon.

After phacoemulsification, raised IOP can occur owing to retained viscoelastic but this is usually self-limiting. ${ }^{20}$ However, the IOP spike after VR surgery can persistent for at least 2 weeks. ${ }^{15}$ It remains unclear whether a raised 
IOP for a short period is damaging, in the absence of pre-existing glaucoma. ${ }^{20}$ Gosse et al ${ }^{21}$ conjecture that the sutureless transconjunctival incisions used for $23 \mathrm{G}$ vitrectomy can act as one-way valves, allowing the release of a small amount of air if the IOP increases significantly. IOP spikes following vitrectomy may become less common as the use of small-gauge vitrectomy instruments becomes more widespread. ${ }^{21,22}$

We have only studied patients who attended our hospital for follow-up on day 1. Around half of patients undergoing vitrectomy do not attend our unit for day-1 follow-up, because some missed their day- 1 visit and a larger population of non-attendees were advised to attend their local eye unit instead for convenience. We have no data about the day- 1 visit for these patients, but we also have no reason to believe that our intervention rate would be any higher if these out-of-area patients had attended our hospital.

There is some selection bias, because we are a tertiary referral centre dealing with complex pathology and we tend to request complex high-risk patients to attend for day-1 review, even if these patients are out-of-area.

Therefore, it is possible that our intervention rate of $3.7 \%$ may well be an overestimate.

The retrospective study design has some advantages over a prospective study when considering intervention rate. It eliminates the possibility of investigator bias that might have influenced decisions on whether to change management at the postoperative visit. ${ }^{18}$ Our results reflect true everyday practice.

Our study is distinct from others in the literature for three reasons. First, we routinely use prophylactic IOP-lowering medication to reduce the risk of an IOP spike. Second, we acknowledge that IOP is not the only factor to be assessed on the first postoperative day and have therefore utilised intervention rate as our primary outcome measure. Third, this is the largest series reported in the published literature of post-vitrectomy intervention rates on day 1 and includes both $20 \mathrm{G}$ and $23 \mathrm{G}$ vitrectomy patients.

Although this study has focussed only on intervention rate, we recognise that next-day postoperative review has some other benefits. It is an opportunity for patient education and allows reinforcement of posturing and postoperative care instructions. It also provides reassurance for patients, especially because, unlike cataract surgery, the immediate postoperative visual acuity may be worse than the preoperative acuity. ${ }^{23}$ It has been suggested that omission of the day-1 review would simply increase the number of emergency attendances in the early postoperative period, before the 2-week review appointment. ${ }^{15}$ For omission of next-day review to be an economically viable and truly desirable alteration to postoperative management, the rate of positive outcomes needs to be maintained, while postoperative complications/events are kept to a minimum. Potential strategies include review before discharge, rapid access for emergency review, prophylactic ocular hypotensive treatment of IOP spikes, and enforcement of shared care principles by other healthcare professionals. ${ }^{18,23,24}$ Ophthalmic nurse practitioners can have a role in reinforcing patient education regarding postoperative care and warning symptoms, reduce patient anxiety, and even review VR surgical outcomes, effectively reducing demands on physicians' time. ${ }^{23}$ A comparative study of first-day postoperative cataract review methods showed that telephone review is the preferred method of followup by patients, and that by telephone any concerning symptoms can effectively be assessed by a nursing staff who can then advise on immediate referral. ${ }^{25}$ Notably, patients did not report any difference in the degree of reassurance they received. ${ }^{25}$

Based on the results of our study, we would recommend that with appropriate safeguarding and streamlining of emergency patients with postoperative complications, routine next-day postoperative review following pars plana vitrectomy can be safely omitted without compromising standards of care and postoperative review at 2 weeks is appropriate and sufficient for most patients.

\section{Summary}

What was known before

- A study published more than 25 years ago found that $52 \%$ of patients undergoing VR required some form of unexpected intervention in the immediate postoperative period. This has defined the standard of care that all patients undergoing VR surgery should receive a postoperative day-1 examination.

- With the advent of quicker, safer vitrectomy as a day case local anaesthetic procedure, the relevance of the day- 1 review remains the subject of debate, and there is a wide variation in practice across the United Kingdom.

- Routine next-day follow-up after cataract surgery was deemed unnecessary when numerous studies found that the intervention rate on day-1 was only $3 \%$.

\section{What this study adds}

- With routine use of prophylactic anti-glaucoma medication in all patients, the intervention rate on day 1 after pars plana vitrectomy, including both 20G and 23G patients, was $3.7 \%$.

- This is the largest study to date of the intervention rate on the first day after pars plana vitrectomy.

- Indication for surgery, type of tamponade, and instrument gauge were not predictive of the need for intervention, although hypotony was less common in patients receiving gas tamponade compared with other forms of tamponade.

- The intervention rate in patients undergoing pars plana vitrectomy is low and routine next-day postoperative review following pars plana vitrectomy can be safely omitted without compromising standards of care. 


\section{Conflict of interest}

The authors declare no conflict of interest.

\section{References}

1 Isernhagen RD, Michels RG, Glaser BM, de Bustros S, Enger C. Hospitalization requirements after vitreoretinal surgery. Arch Ophthalmol 1988; 106(6): 767-770.

2 Costen MT, Newsom RS, Wainwright AC, Luff AJ, Canning CR. Expanding role of local anaesthesia in vitreoretinal surgery. Eye (Lond) 2005; 19(7): 755-761.

3 Newsom R, Luff A, Wainwright C, Canning C. UK survey of attitudes to local anaesthesia for vitreoretinal surgery. Eye (Lond) 2001; 15(Pt 6): 708-711.

4 Newsom RSB, Wainwright AC, Canning CR. Local anaesthesia for 1221 vitreoretinal procedures. $\mathrm{Br} \mathrm{J} \mathrm{Ophthalmol}$ 2001; 85(2): 225-227.

5 Wong R, Gupta B, Williamson TH, Laidlaw DA. Day 1 postoperative intraocular pressure spike in vitreoretinal surgery (VDOP1). Acta Ophthalmol 2011; 89(4): 365-368.

6 El-Amir AN, Keenan TD, Abu-Bakra M, Tanner V, Yeates D, Goldacre MJ. Trends in rates of retinal surgery in England from 1968 to 2004: studies of hospital statistics. $\mathrm{Br} \mathrm{J}$ Ophthalmol 2009; 93(12): 1585-1590.

7 Admitted Patient Care - England. Hospital Episode Statistics. Available at http://www.hscic.gov.uk/searchcatalogue? productid $=9161 \&$ topics $=1 \% 2 \mathrm{fHospital}+$ care $\% 2$ fInpatient +admissions\&sort $=$ Relevance\&size $=10 \&$ page $=1$-top .

8 Fernando A, Gorman C. Stopping the 1st post operative day review following vitrectomy: a BEAVRS survey. Annual Meeting of the British \& Eire Association of Vitreoretinal Surgeons. Canterbury: United Kingdom, 2011.

9 Vitrectomy with silicone oil or sulfur hexafluoride gas in eyes with severe proliferative vitreoretinopathy: results of a randomized clinical trial. Silicone Study Report 1. Arch Ophthalmol 1992; 110(6): 770-779.

10 Chang S, Lincoff HA, Coleman DJ, Fuchs W, Farber ME. Perfluorocarbon gases in vitreous surgery. Ophthalmology 1985; 92(5): 651-656.

11 Vitrectomy with silicone oil or perfluoropropane gas in eyes with severe proliferative vitreoretinopathy: results of a randomized clinical trial. Silicone Study Report 2. Arch Ophthalmol 1992; 110(6): 780-792.

12 Abrams GW, Swanson DE, Sabates WI, Goldman AI. The results of sulfur hexafluoride gas in vitreous surgery. Am J Ophthalmol 1982; 94(2): 165-171.
13 Sciscio A, Casswell AG. Effectiveness of apraclonidine 1\% in preventing intraocular pressure rise following macular hole surgery. Br J Ophthalmol 2001; 85(2): 164-168.

14 Benz MS, Escalona-Benz EM, Murray TG, Eifrig CW, Yoder DM, Moore JK et al. Immediate postoperative use of a topical agent to prevent intraocular pressure elevation after pars plana vitrectomy with gas tamponade. Arch Ophthalmol 2004; 122(5): 705-709.

15 Lee R, Charisis S, Orr G. To see, or not to see, that is the question. Annual Meeting of the British \& Eire Association of Vitreoretinal Surgeons. Bristol: United Kingdom, 2013.

16 Cochrane T, Tanner V. Day case VR surgery without next day review, is it safe? Annual Meeting of the British \& Eire Association of Vitreoretinal Surgeons. Bristol: United Kingdom, 2013.

17 Russell-Hermanns DS, Braniff C, Taylor C, Woodcock M. Prospective study investigating intra-ocular pressure (lOP) elevation after vitreoretinal surgery. Annual Meeting of the British \& Eire Association of Vitreoretinal Surgeons. Bristol: United Kingdom, 2013.

18 Allan BD, Baer RM, Heyworth P, Duguid IG, Dart JK. Conventional routine clinical review may not be necessary after uncomplicated phacoemulsification. $\mathrm{Br} J$ Ophthalmol 1997; 81(7): 548-550.

19 Whitefield L, Crowston J, Little BC. First day follow up for routine phacoemulsification? Br J Ophthalmol 1996; 80(2): 148-150.

20 Tan JH, Newman DK, Klunker C, Watts SE, Burton RL. Phacoemulsification cataract surgery: is routine review necessary on the first post-operative day? Eye (Lond) 2000; 14 (Pt 1): 53-55.

21 Gosse E, Newsom R, Hall P, Lochhead J. Changes in day 1 post-operative intraocular pressure following sutureless 23-gauge and conventional 20-gauge pars plana vitrectomy. Open Ophthalmol J 2013; 7: 42-47.

22 Misra A, Ho-Yen G, Burton RL. 23-gauge sutureless vitrectomy and 20-gauge vitrectomy: a case series comparison. Eye (Lond) 2009; 23(5): 1187-1191.

23 Herbert EN, Gibbons H, Bell J, Hughes DS, Flanagan DW. Complications of phacoemulsification on the first postoperative day: can follow-up be safely changed? J Cataract Refract Surg 1999; 25(7): 985-988.

24 Alwitry A, Rotchford A, Gardner I. First day review after uncomplicated phacoemulsification: is it necessary? Eur J Ophthalmol 2006; 16(4): 554-559.

25 Mandal K, Dodds SG, Hildreth A, Fraser SG, Steel DH. Comparative study of first-day postoperative cataract review methods. J Cataract Refract Surg 2004; 30: 1966-1971. 